\title{
RETENCIONES CON TIPO DE CAMBIO FLEXIBLE $^{\circ}$
}

\author{
EXPORT TAXES: THE CASE OF A FLOATING \\ EXCHANGE RATE
}

Víctor A. Beker*

enviado 18 de junio de 2019 - aceptado 24 de junio de 2019

\begin{abstract}
Resumen
Habitualmente, cuando se analiza el efecto que tiene la implantación de retenciones a las exportaciones se lo hace suponiendo implícitamente que existe un tipo de cambio fijo. En esta nota se analiza el caso en que el tipo de cambio es flexible. Se demuestra que la retención se traslada en mayor o menor medida al tipo de cambio. Solo en los casos extremos en que la demanda fuera infinitamente elástica o que la oferta fuera perfectamente inelástica la retención recaerá totalmente sobre los oferentes.

Código JEL: F10, F13, H25.

Palabras clave: retenciones, tipo de cambio fijo, tipo de cambio flexible, precio interno, precio internacional.
\end{abstract}

- Beker V. A. (2019). Retenciones con tipo de cambio flexible. Estudios económicos, 36 (73), 191-197.

* Universidad de Belgrano y Unversidad de Buenos Aires. Correo electrónico: victor. beker@ub.edu.ar 


\begin{abstract}
Usually, when analyzing the effect of export taxes it is implicitly assumed that there is a fixed exchange rate. This note discusses the case in which the exchange rate is flexible. It shows that in such a case the export tax is to a greater or lesser extent passed on to the exchange rate. Only in the extreme cases when demand is infinitely elastic or supply is perfectly inelastic the tax burden will fall entirely on the suppliers.
\end{abstract}

JEL Code: F10, F13, H25.

Keywords: export taxes, fixed exchange rate, floating exchange rate, domestic price, international price. 


\section{INTRODUCCIÓN}

Habitualmente, cuando se analiza el efecto que tiene la implantación de aranceles a las importaciones o de retenciones a las exportaciones se lo hace suponiendo implícitamente que existe un tipo de cambio fijo.

Sin embargo, las conclusiones que se obtienen dejan de ser válidas cuando se postula la existencia de tipo de cambio flexible.

\section{Retenciones con tipo de cambio fijo}

consideremos que $\mathrm{D}_{\mathrm{n}}$ y $\mathrm{O}_{\mathrm{n}}$ son las curvas de demanda y oferta y oferta nacionales, respectivamente, de un bien exportado (la soja, por ejemplo). $\mathrm{P}_{\mathrm{o}}$ sería el precio de equilibrio sin comercio internacional y $\mathrm{Q}_{0}$ la cantidad que se intercambiaría a dicho precio.

Supongamos que el precio internacional es $\mathrm{P}_{1}$. A dicho precio ${ }^{1}, O Q_{n}$ es la cantidad que absorbe la demanda local mientras que $Q_{n} Q_{1}$ es la cantidad exportada (figura 1).

Figura 1. Situación sin retenciones

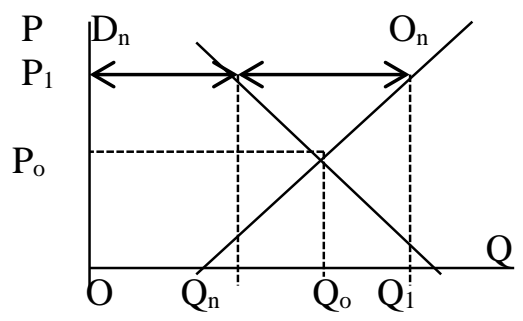

Fuente: elaboración propia.

1 Estrictamente, $\mathrm{P}_{1}$ es el precio internacional en dólares multiplicado por el tipo de cambio. 
Supongamos que se establece una retención a las exportaciones. Ello hace que el precio que perciba el oferente sea ahora el precio internacional menos la cuantía de la retención. Representamos la nueva situación en la figura 2.

Figura 2. Situación con retenciones

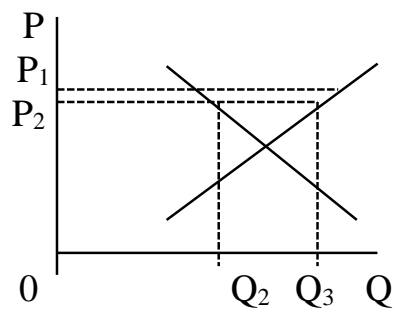

Fuente: elaboración propia.

En la nueva situación, la oferta nacional se ha reducido a $\mathrm{Q}_{3}$, la exportación cae a $\mathrm{Q}_{2} \mathrm{Q}_{3}$, el precio internacional sigue siendo $\mathrm{P}_{1}$ pero el oferente recibe $\mathrm{P}_{2}$ y la diferencia es la retención que percibe el Estado por cada unidad exportada. El consumo se incrementa porque ahora el precio interno $\mathrm{P}_{2}$ es inferior al precio internacional $\mathrm{P}_{1}$.

En resumen, la implantación de la retención reduce la producción nacional, incrementa el consumo y ambos efectos generan un menor nivel de exportaciones. El fisco se hace de una nueva fuente de ingresos.

\section{Retenciones con tipo de cambio flexible}

Si el tipo de cambio deja de ser fijo y pasa a ser determinado por la oferta y demanda de divisas, debe analizarse qué efecto puede tener el establecimiento de retenciones sobre el mercado cambiario.

Por razones de simplicidad, supongamos que la demanda de divisas (dólares) tiene como único objetivo el pago de importaciones mientras que la oferta proviene exclusivamente de las ventas que realizan los exportadores. Por la misma razón, vamos a suponer que se establece una retención sobre las exportaciones de 
suma fija, aunque el análisis se podría extender fácilmente al caso de una retención porcentual.

En la figura 3, sea $\mathrm{D}^{\mathrm{d}}$ la función de demanda de dólares y $\mathrm{O}^{\mathrm{d}}$ su oferta, en ausencia de retenciones $\mathrm{T}_{0}$ será el tipo de cambio de equilibrio. La implantación de retenciones a las exportaciones desplazará la curva de oferta hacia la izquierda. $\mathrm{O}^{\mathrm{d} 1}$ es la nueva curva de oferta. El nuevo tipo de cambio de equilibrio será $\mathrm{T}_{1}$. El efecto de las retenciones es aumentar el tipo de cambio.

Figura 3. Situación con retenciones
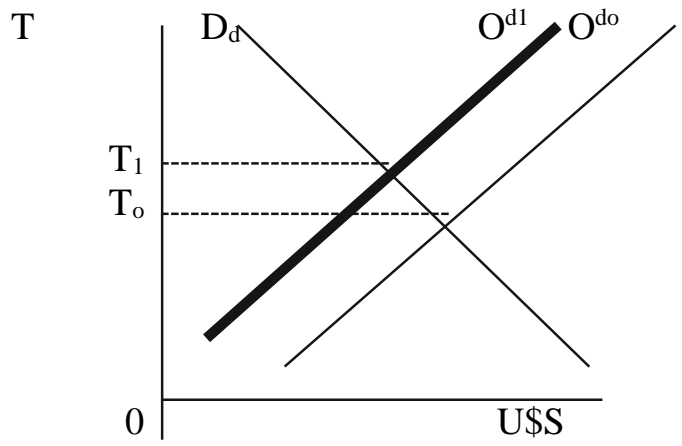

Fuente: elaboración propia.

Dicho de otra manera, el importe de la retención se traslada al tipo de cambio de equilibrio en mayor o menor medida dependiendo de las elasticidades de las curvas de demanda y oferta de divisas. Cuanto más inelástica la demanda, mayor es el traslado al tipo de cambio; en el límite, si la demanda fuera totalmente inelástica la retención se trasladaría totalmente y el monto exportado no se modificaría. La retención opera como un arancel sobre las importaciones. En el extremo opuesto, si la demanda fuera infinitamente elástica, el tipo de cambio no se modificaría y se reduciría la cantidad exportada como se ilustra en la figura 4. 
Figura 4. Situación con retenciones

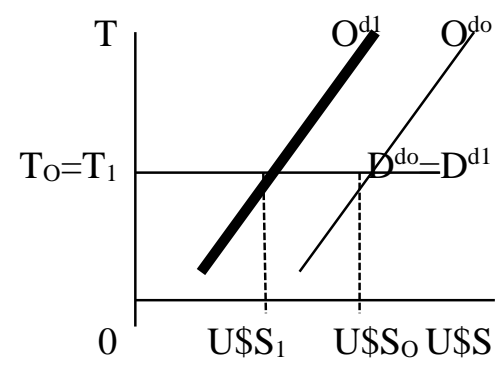

Fuente: elaboración propia.

Similar análisis puede hacerse respecto a la elasticidad de la curva de oferta: cuanto más inelástica sea la curva de la oferta, menor es el traslado de la retención sobre el tipo de cambio; si la oferta fuera perfectamente inelástica, la retención no se trasladaría en absoluto, sería totalmente absorbida por los oferentes y no se modificaría la cantidad exportada, como se ilustra en la figura 5.

Figura 5. Situación con retenciones

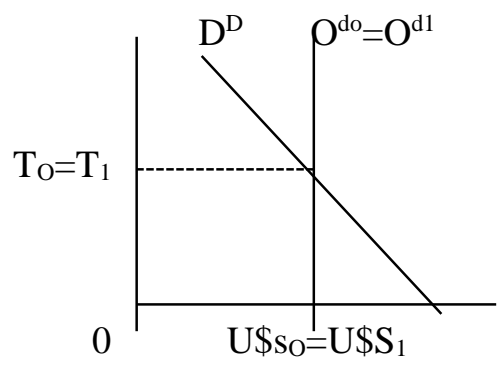

Fuente: elaboración propia.

En cambio, si la curva de oferta fuera perfectamente elástica, la retención se trasladaría totalmente al tipo de cambio y se reduciría la cantidad exportada. 
En síntesis, cuanto más inelástica la curva de demanda y más elástica la de oferta, mayor es el traslado de la retención al tipo de cambio y menor el ajuste en la cantidad exportada.

\section{CONCLUSIÓN}

Cuando el tipo de cambio es fijo y se establece una retención sobre las exportaciones, la misma recae totalmente sobre los oferentes que perciben el precio internacional del producto exportado menos el importe de la retención. No necesariamente ocurre así cuando existe un tipo de cambio flexible.

En este caso, la retención se traslada en mayor o menor medida al tipo de cambio, dependiendo de las elasticidades de las curvas de demanda y oferta de divisas. Solo en los casos extremos en que la demanda fuera infinitamente elástica o que la oferta fuera perfectamente inelástica la retención recaerá totalmente sobre los oferentes. Fuera de estos casos extremos, la retención recaerá total o parcialmente sobre los importadores a través de su traslado al tipo de cambio de equilibrio.

(C) 2019 por los autores; licencia otorgada a la revista Estudios económicos. Este artículo es de acceso abierto y distribuido bajo los términos y condiciones de una licencia Atribución-No Comercial 3.0 Unported (CC BY-NC 3.0) de Creative Commons. Para ver una copia de esta licencia, visite http://creativecommons.org/ licenses/by-nc/3.0/ 
